\title{
Spontaneous idiopathic aneurysm of the inferior vena cava
}

\author{
Benoit Cosset $^{1}$, Daniel Grinberg ${ }^{1}$, Marine BORDET ${ }^{1}$, and Fadi FARHAT ${ }^{1}$ \\ ${ }^{1}$ Hopital Cardio-vasculaire et Pneumologique Louis Pradel
}

April 30, 2021

\begin{abstract}
Inferior vena cava (IVC) aneurysms are uncommon. It is only 54 cases reported in English medical literature. Venous aneurysms are associated with long standing systemic venous hypertension, trauma, inflammatory processes or congenital malformation. Herein we report a case of spontaneous aneurysm of IVC which was not associated with any of the above mentioned predisposing factors.
\end{abstract}

\section{Spontaneous Aneurysm of The Inferior Vena Cava}

Benoit COSSET ${ }^{1}$, MD, Daniel GRINBERG, MD,PhD, Marine BORDET ${ }^{2}$, Fadi FARHAT ${ }^{1}$, MD,PhD

1 Department of cardiovascular Surgery B, Louis Pradel Hospital, Bron, France

2 Department of vascular Surgery, Louis Pradel Hospital, Bron, France

Key words : inferior vena cava, spontaneous, aneurysm, surgery

Corresponding author:

Professor Fadi Farhat, MD, PhD

Department of Cardiovascular Surgery B

Hôpital Cardiovasculaire et Pneumologique Louis Pradel, Université Claude Bernard, INSERM CARMEN. 28, avenue du doyen Lépine, 69677, BRON Cedex, France.

Tel: 33 (0)4 723575 29, Fax: 33 (0)4 7212 95, E-mail:fadi.farhat@chu-lyon.fr

\begin{abstract}
Inferior vena cava (IVC) aneurysms are uncommon. It is only 54 cases reported in English medical literature. Venous aneurysms are associated with long standing systemic venous hypertension, trauma, inflammatory processes or congenital malformation. Herein we report a case of spontaneous aneurysm of IVC which was not associated with any of the above mentioned predisposing factors.
\end{abstract}

Data Availability statement : all data are available

Informed consent was obtained from the patient

Ethic approval was obtained from local board

Funding : none

Conflict of interest : none

Case report 
A 31-year-old male was referred to our hospital for further evaluation of a retroperitoneal cyst which was found incidentally by abdominal ultrasonography during evaluation of abdominal pain and diarrhea about two years ago.

The patient presented with mild lower limbs swelling and venous insufficiency. Duplex ultrasonography displayed bilateral deep femoral vein thrombosis. Thoracic CT angiography was performed but did not find any abnormalities. The retroperitoneal cyst was further explored by MRI which displayed a huge $(9 \times 7 \times 7.5 \mathrm{~cm})$ aneurysm of infrarenal IVC (figure 1). Laboratory examinations including coagulation profile were normal.

Due to the risk of aneurysmal thrombosis and rupture, surgical intervention was indicated. The operation was started by median laparotomy using mesenteric root approach, infrarenal aorta was freed. Dissection continued, the proximal and distal control of vena cava at infrarenal and iliac veins junction level was obtained. The aneurysm, which was located posterior to the right kidney, was also dissected free from adjacent tissues. The patient was heparinized and the aorta was clamped to decrease venous return to IVC. The IVC proximally at infrarenal level and distally at the junction of iliac veins excluding the aneurysmal area was clamped (figure 2 upper).

The IVC was opened longitudinally and a huge aneurysm was found while the walls of vena cava were normal (figure 2 under). Afterwards the aneurysm was resected and two specimens sent for bacteriology and histopathology respectively. IVC was closed by continuous non absorbable sutures and after de-airing clamps were also released, no leakage was seen. An active drain was left in the aneurysmal lodge (and was latterly removed on second post-operative day). Peritoneum and abdominal wall were closed.

Histopathological examination found no sign of inflammation but slight modifications of venous wall with a miner interstitial fibrosis otherwise containing endothelium and a regular tunica muscularis which was surrounded by adventitia. Bacteriologic examination was also sterile after two weeks of incubation period.

Post operatively the patient recovered without any complications and was discharged on 8th postoperative day with only aspirin. Doppler ultrasonography was performed on 45th postoperative day and showed good flow in IVC and in femoral veins.

\section{Discussion}

IVC aneurysms are uncommon. It is only 54 cases reported in English medical literature (1).The etiologies of IVC aneurysms remain unclear, but in some acquired cases of IVC aneurysms trauma and inflammatory processes are involved as causative factor. In addition, longstanding systemic venous hypertension secondary to right side heart failure, tricuspid valve lesions, cardiomyopathy, and constrictive pericarditis may also contribute to the formation of IVC aneurysms. Moreover, IVC aneurysms have been associated with embryogenic malformation such as tetralogy of Fallot, azygous agenesis, membranous IVC obstruction and left side IVC $(1,2,4)$.

An aneurysm of IVC may present with the symptoms related to complications such as thromboembolism or IVC syndrome or may have some nonspecific abdominal or back pain. Although, uncomplicated aneurysm of IVC may have no symptoms $(1,2,4,6)$. In our case, it was the diarrhea, abdominal pain and lower extremity venous insufficiency which lead us to the diagnosis. Imaging studies such as MRI angiography, CT angiography and venography are diagnostic $(2,3,4)$. Duplex ultrasonography is also helpful. According to Gradman and Steinberg classification of IVC aneurysms our case is a type III IVC aneurysm (5). Type II-IV IVC aneurysms have high rate of complications such as thromboembolism and rupture $(1,2,3)$.

Management of IVC aneurysms entirely depends on their nature and presence of complications $(1,3,6)$. In our case we proceeded to perform surgical resection of aneurysm because there was a high risk of complications and there was a low probability of surgical complications. The endovascular approach was discussed but was rejected because of the risk of IVC rupture in case of huge oversizing of the endograft (in order to avoid any endoleak). Moreover, a septic cause of the pseudo aneurysm was not excluded.

In our case we did not find any past history of trauma. Additionally, histopathologic and bacteriologic 
examinations of aneurysmal tissue biopsy did not find any particular abnormality in favor of inflammatory process and complementary examinations did not find any associated congenital malformation. Therefore we presumed our case as spontaneous (idiopathic).

\section{Conclusion}

While approaching a patient with IVC aneurysm or diagnosing as one, beside all other etiologic factors, a spontaneous case should also be taken into consideration. Surgical management should be proposed in case of an estimated high risk of rupture, and also to have an etiologic diagnosis if possible.

\section{Figure 1}

MRI image of IVC aneurysm

Figure 2

Above: intra operative view of the venous aneurysm. 1.Aorta, 2.IVC, 3.IVC aneurysm

Under: 1.IVC aneurysm opened longitudinally

\section{References}

1. Monterro-Baker M, Branco BC, Leon LL Jr, Labropoulos N, Echeverria A, Mills JL Sr. Management of inferior vena cava aneurysm. J Cardiovasc Surg 2015;56:769-74.

2. Elliot A, Henn A, Pamuklar E, Rivero H, Hyslop WB, Semelka RC, Burke CT . Aneurysm of inferior vena cava: case report. Abdom Imaging 2006;31:457-60.

3. Davidovic L, Dragas M, Bozic M, Takac D. Aneurysm of the inferior vena cava: case report and review of the literature. Phlebology 2008;23:184-8.

4. Suresh KR, Vivekanand V, Vishnu M, Sumanth R. Aneurysm of the inferior vena cava. J Vasc Surg $2007 ; 45: 844$

5. Gradman W, Steinberg F. Aneurysm of the inferior vena cava: Case Report and Review of the Literature. Ann Vasc Surg 1993;7:347-53. 


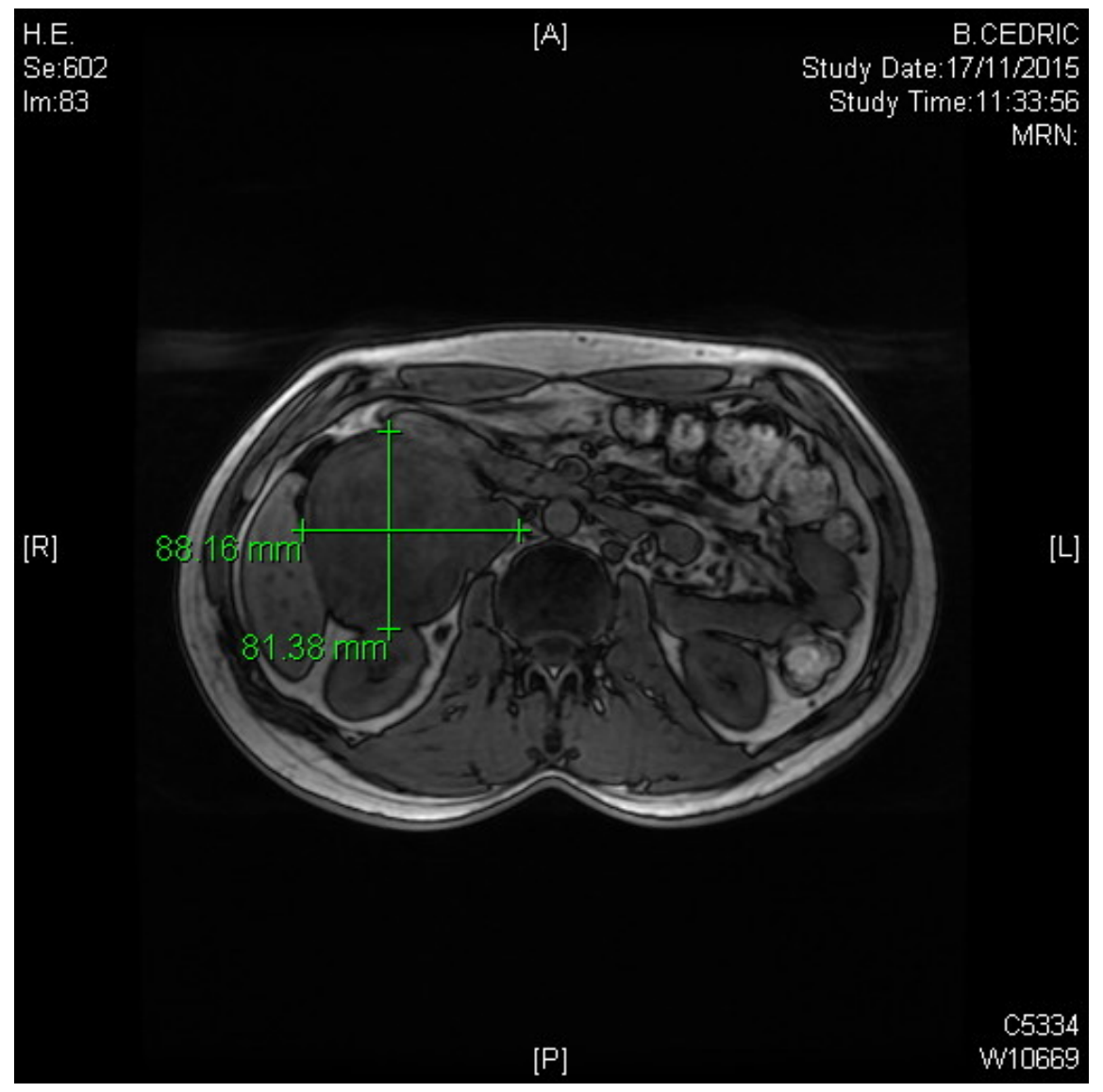




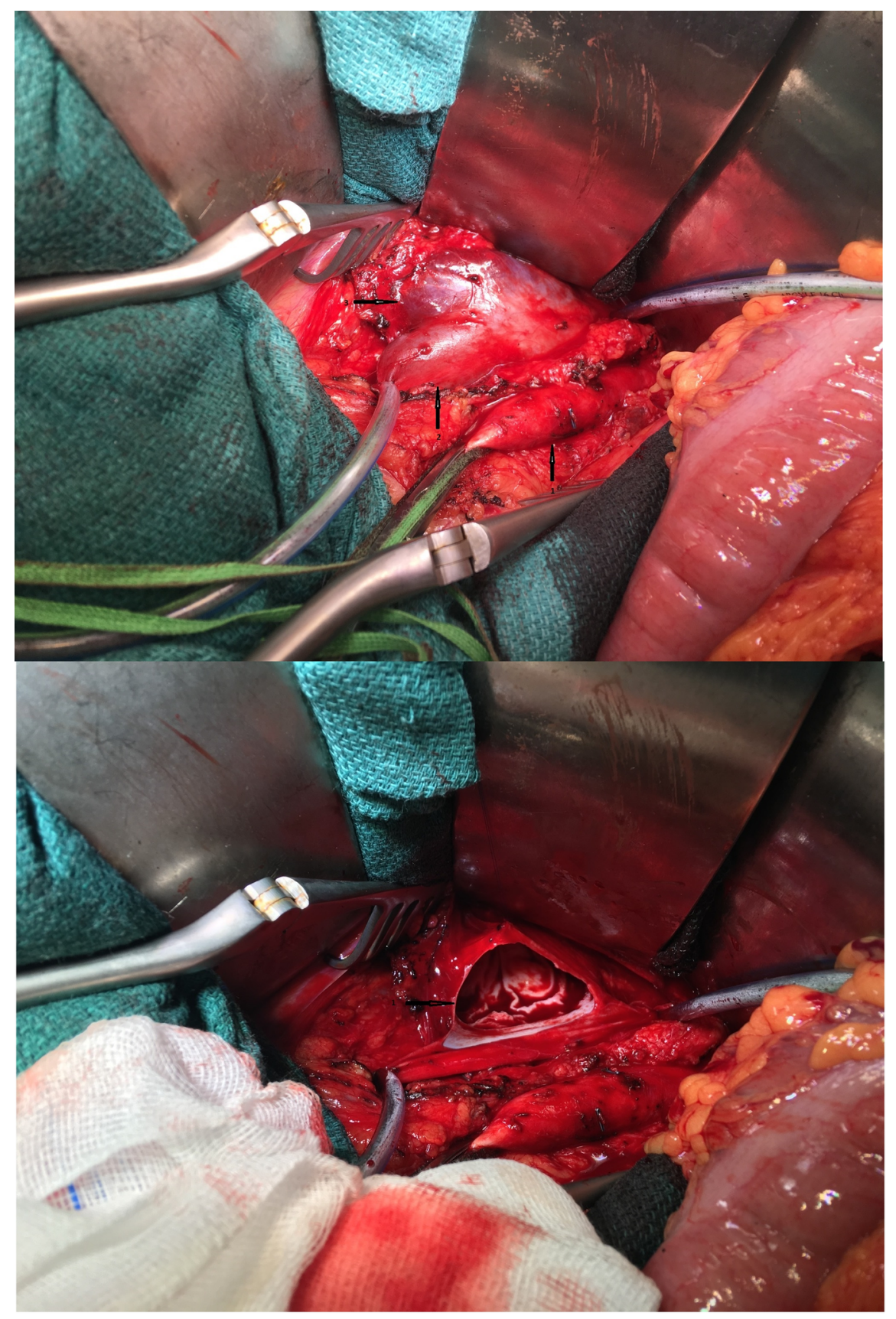

